\title{
A Single Amino Acid Mutation in the Plum pox virus Helper Component-Proteinase Gene Abolishes Both Synergistic and RNA Silencing Suppression Activities
}

\author{
Pablo González-Jara, Felix A. Atencio, Belén Martínez-García, Daniel Barajas, \\ Francisco Tenllado, and José Ramón Díaz-Ruíz
}

Departamento de Biología de Plantas, Centro de Investigaciones Biológicas, CSIC, Av. Ramiro de Maeztu 9, 28040 Madrid, Spain. Accepted for publication 5 April 2005.

\begin{abstract}
González-Jara, P., Atencio, F. A., Martínez-García, B., Barajas, D., Tenllado, F., and Díaz-Ruíz, J. R. 2005. A single amino acid mutation in the Plum pox virus helper component-proteinase gene abolishes both synergistic and RNA silencing suppression activities. Phytopathology 95:894-901.

The effects on symptom expression of single amino acid mutations in the central region of the Plum pox virus (PPV) helper component-proteinase (HC-Pro) gene were analyzed in Nicotiana benthamiana using Potato virus $X$ (PVX) recombinant viruses. PVX recombinant virus expressing the wild-type variant of PPV HC-Pro induced the expected enhancement of PVX pathogenicity, manifested as necrosis and plant death. Recombinant virus expressing a variant of PPV HC-Pro containing a single point

tant showed no such activity. These results indicate that a unique point mutation in PPV HC-Pro impaired its ability to suppress RNA silencing and abolished its capacity to induce synergism, and clearly shows for the first time the link between these two functions in potyvirus HC-Pro. Additionally, we compared the effects on virus accumulation in $N$. benthamiana plants infected with either the PVX recombinant constructs or with native viruses in double infection experiments. PVX $(+)$ and $(-)$ strand genomic RNA accumulated at similar levels in plants infected with PVX recombinants, leading to an increase in PVX pathology, compared with plants infected with PVX alone. This finding confirms that the enhancement of pathogenicity associated with synergistic interaction is not a consequence of more efficient PVX replication due to RNA silencing suppression by PPV HC-Pro.
\end{abstract} mutation $\left(\mathrm{HCL}_{134} \mathrm{H}\right)$ was unable to induce this synergistic phenotype. The RNA silencing suppressor activity of PPV HC-Pro was demonstrated in a transient silencing suppression assay. In contrast, the $\mathrm{HCL}_{134} \mathrm{H}$ mu-
Additional keywords: posttranscriptional gene silencing.
Diseases caused by the interaction of two different viruses are common in higher plants and often result in the intensification of symptom expression and virus accumulation, a phenomenon known as synergism (9). The synergistic interaction of Potato virus $X$ (PVX) with a number of potyviruses, including Potato virus $Y$ (PVY) or Tobacco etch virus (TEV), have been studied in detail in tobacco (Nicotiana tabacum) $(7,20,26,27)$ and, to a lesser extent, in $N$. benthamiana (6). Double infection of tobacco plants with PVX and PVY, TEV, or other potyviruses induces a disease characterized by the dramatic enhancement of symptoms in systemically infected leaves which become necrotic. Further, a corresponding increase in PVX levels (up to 10-fold) is also seen compared with singly infected plants. In contrast, the accumulation of PVX does not increase greatly in $N$. benthamiana when doubly infected with PVX and either PVY or TEV, despite the enhancement of symptoms leading to systemic necrosis and plant death. This indicates that the differences between the ways in which synergism is manifested in $N$. benthamiana and tobacco are hostdependent (6). Additionally, in transgenic tobacco plants it has been shown that potyvirus replication is not required for synergism to occur, but only the expression of the 5'-proximal sequences encoding P1 and the helper component-proteinase (HC-

Corresponding author: J. R. Díaz-Ruíz; E-mail address: jrdiazruiz@cib.csic.es

* The $\boldsymbol{e}$-Xtra logo stands for "electronic extra" and indicates that the online version contains supplemental material not included in the print edition. Figures 1 and 5 are in color online.

DOI: 10.1094/PHYTO-95-0894

(C) 2005 The American Phytopathological Society
Pro) proteins (27). Further, the expression of the HC-Pro protein by a PVX vector is sufficient to induce the increase of PVX pathogenicity in $N$. benthamiana $(20,22)$.

Besides its role in synergism and trans-activation of replication in heterologous viruses, HC-Pro fulfils many biological functions in the potyviral life cycle. In particular, the central region of the protein, where the determinants for synergism map, is also involved in genomic amplification, cell-to-cell and long-distance movement, symptom expression, binding of nucleic acids, suppression of posttranscriptional gene silencing (PTGS), and virusinduced gene silencing (summarized in 22). PTGS is one of the many manifestations of RNA silencing, a sophisticated mechanism by which the suppression of gene expression is achieved through nucleotide-sequence-specific interactions based on RNA. In plants, a function of PTGS is to act as an adaptive defense response to DNA and RNA viruses (28). As a counter defense, many plant viruses encode proteins that suppress PTGS, such as HC-Pro in PVY and TEV, and p25 in PVX (21). The same mutations that affect the systemic movement and replication maintenance functions of HC-Pro also disable the protein for PTGS suppression activity (11). The fact that other mutations of the central region of HC-Pro impair the protein for synergism suggests that this function and the suppression of gene silencing are related (24). Therefore, the inhibition of this plant defense mechanism by HC-Pro would allow viruses to accumulate beyond the normal host-imposed limits and induce more severe disease (17, 29). Recent findings, however, suggest that disease related to PVX-potyvirus-associated synergism may involve interference with multiple RNA silencing pathways. In addition to inhibiting the antiviral silencing response, Turnip mosaic virus HC-Pro interferes with micro (mi)RNA-guided mRNA cleavage (another 
manifestation of RNA silencing) causing developmental abnormalities in transgenic Arabidopsis thaliana $(5,12)$.

During a series of studies on aphid transmissibility of two Plum pox virus (PPV) isolates, we cloned several variants of the $\mathrm{HC}$ Pro gene from PPV. In the present work we introduced two of these variants into a PVX-based vector to determine the effects of different mutations on the HC-Pro-induced enhancement of PVX pathogenicity. We also compared the effects on virus accumulation after $N$. benthamiana plants were infected with either of these PVX recombinant constructs or with native viruses in double infection experiments.

\section{MATERIALS AND METHODS}

Recombinant viruses and plant inoculation. The two Spanish PPV isolates used in this work, 5.15 and 3.3, have been previously described (16). $N$. benthamiana plants infected with either isolate showed undistinguishable symptoms, typically mild vein clearing and systemic, chlorotic mosaic. However, aphid transmission experiments showed that isolate 5.15 was transmitted from infected plants, whereas isolate 3.3 was not. In the course of studies to determine whether the lack of transmission of isolate 3.3 was due to an alteration in the HC-Pro protein, a series of cDNA clones of the PPV HC-Pro gene were obtained by means of reverse transcription-polymerase chain reaction (RT-PCR) with RNA extracted from $N$. benthamiana plants infected with either of the PPV isolates. In the present work, two cloned variants of the HC-Pro gene were used, p3.3HCwt and p5.15HCmut. pP2C2S402, a cDNA clone of PVX derived from pP2C2S (2), was used as a vector for expression of PPV HC-Pro in plants. The HC-Pro sequences contained in $\mathrm{p} 3.3 \mathrm{HCwt}$ and $\mathrm{p} 5.15 \mathrm{HCmut}$ were PCRamplified and individually cloned into the PVX vector, under the control of the duplicated PVX coat protein $(\mathrm{CP})$ promoter, to give pPVX-HCwt and pPVX-HCmut, respectively. The upstream primer (HC1) was 5'-CAACGGCCGATGTACTCTGACCCA$G G C-3^{\prime}$ (nucleotides 1068 to 1082 of the PPV sequence in italics) (13), which provides an EagI site (underlined) and a translational start codon (bold). The downstream primer (HC2) was 5'GTTGTCGACTTATGCAACCAGGTATGT-3' (nucleotides complementary to 2430 to 2444 in italics), which introduces an SalI site (underlined) and a stop codon (bold).

The two point changes in the HC-Pro central region of pPVXHCmut (Table 1) were reproduced individually in pPVX-HCwt to give two new mutant HC-Pro encoding PVX derivatives: pPVX$\mathrm{HCL}_{134} \mathrm{H}$ and $\mathrm{pPVX}-\mathrm{HCI}_{272} \mathrm{~V}$. To this end, a recombinant PCR technique was used as described in Higuchi et al. (8), employing pPVX-HCwt as the template. The internal mutagenic primers used to produce mutation $\mathrm{L}_{134} \mathrm{H}$ were $5^{\prime}$-TTTGTTGTGATGGG-3' (nucleotides complementary to 1465 to 1478 ) and $5^{\prime}$-TTCCCATCACAACAAAATC-3' (nucleotides 1463 to 1481). The internal mutagenic primers used to produce $\mathrm{I}_{272} \mathrm{~V}$ were $5^{\prime}$-AATGAACGCACTCTTCAC $-3^{\prime}$ (nucleotides complementary to 1873 to 1890 ) and 5'-AAGAGTGCGTTCATTTTG-3' (nucleotides 1876 to 1893). The nucleotide alterations responsible for the amino acid changes are shown in bold. Primers HC1 and/or HC2 were employed in each PCR as external primers. Each of the two new amplified HC-Pro mutant sequences were cloned into pBluescript $\mathrm{KS}(+)$ and finally transferred to PVX vector by double EagI/SalI digestion. The presence of the nucleotide substitutions and the lack of undesired changes in the HC-Pro sequence were confirmed by sequencing. Plasmids and cDNAs were manipulated according to standard techniques (23).

Full-length cDNA clones containing PVX derivatives were linearized with SpeI before use in transcription reactions as previously described (4). Ten-microliter volumes were inoculated into two leaves of each plant dusted with celite. Dual inoculation experiments with PVX and PPV isolate 5.15 were performed essentially as described in Gonzalez-Jara et al. (6). N. benthamiana plants were grown in a controlled temperature chamber at 22 to $25^{\circ} \mathrm{C}$. A $16-\mathrm{h} / 8$-h light/dark cycle was established.

Transient silencing suppression assay. The complete HC-Pro coding region was amplified by PCR from pPVX-HCwt with the upstream primer 5'-CGCACCATGGACTCTGACCCTGGCAA-3' (nucleotides 1071 to 1084 in italics, NcoI site underlined, start codon in bold) and the downstream primer 5'-GCCATCTAGACTTATCCAACCAGGTATGT-3' (nucleotides complementary to 2430 to 2444 in italics, $X b a$ I site underlined, stop codon in bold). The PCR-amplified fragment was cloned into the plasmid pAVA393 (provided by A. G. von Arnim, University of Tennessee) linearized with $N c o I$ and $X b a I$. The expression cassette from the pAVA393-based construct was SalI/XmaI-excised and inserted into the plant transformation vector pCAMBIA2300 to provide the pCAM-HCwt plasmid. pPVX-HCmut cDNA was digested with AleI and AatII (nucleotides 1192 and 1771, respectively), and the fragment carrying the mutation $\mathrm{L}_{134} \mathrm{H}$ was inserted into similarly digested pCAM-HCwt. The plasmid obtained was named pCAM- $\mathrm{HCL}_{134} \mathrm{H}$. Both this and pCAM-HCwt were introduced separately into Agrobacterium tumefaciens LBA4404 by heat shock.

A. tumefaciens GV2260 containing the plasmid pSLJ-GFP (10) was provided by J. C. Carrington (Oregon State University). A. tumefaciens carrying pCAMBIA1305.1 containing a gene encoding $\beta$-glucuronidase (GUS) was used as negative control in silencing suppression assays.

Combinations of Agrobacterium cultures carrying pSLJ-GFP, pCAM-HCwt, pCAM- $\mathrm{HCL}_{134} \mathrm{H}$, or pCAMBIA1305.1 were infiltrated into leaves as previously described (10). Leaves were photographed 9 days later using a UV transilluminator and a Kodak DC290 digital camera with a yellow filter.

RNA and protein gel blot analysis. At 10 days postinoculation (dpi), total nucleic acids were isolated from the second systemically infected half-leaf of plants infected with the recombinant viruses as previously described (15). RNA samples were electrophoresed on $1 \%$ (wt/vol) agarose formaldehyde gels (23) and transferred to positively charged nylon membranes. Membrane hybridization was carried out overnight at $65^{\circ} \mathrm{C}$ using digoxigenin (DIG)-labeled riboprobes according to the manufacturer's instructions (Roche Molecular Biochemicals, Mannheim, Germany). Viral RNAs (plus- and minus-sense strands) were detected by hybridization with appropriate probes corresponding to either PVX CP (6) or PPV HC-Pro sequences. The PPV HC-Pro probe was obtained by digestion of pT3T7-HC with XbaI followed by transcription with T7 RNA polymerase. pT3T7-HC was obtained by amplifying the PPV HC-Pro coding sequence by RT-PCR and cloning it into the pT3T7 vector. The upstream primer was 5'AAATCTAGAATGTACTCTGACCCAGGC-3' (nucleotides 1068 to 1082 in italics), which introduced an $X b a \mathrm{I}$ site (underlined). The downstream primer was 5'-GAAGGATCCTTATCCAACCAGGTATGT-3' (nucleotides complementary to 2444 to 2430 in italics), which introduced a BamHI site (underlined).

RNA extraction for the analysis of transgene mRNA was performed with the RNeasy Plant Mini Kit (Qiagen, Chatsworth,

TABLE 1. Amino acid substitutions detected within the helper componentproteinase (HC-Pro) sequence of p5.15HCmut

\begin{tabular}{lllr}
\hline p3.3HCwt $^{\mathrm{a}}$ & p5.15HCmut & \multicolumn{1}{c}{ Region } & Position $^{\mathrm{b}^{\circ}}$ \\
\hline Val (HNP) & Ala (HNP) & N-terminal & $39(347)$ \\
Cys (POU) & Ser (POU) & N-terminal & $50(358)$ \\
Leu (HNP) & His (+) & Central & $134(442)$ \\
IIe (HNP) & Val (HNP) & Central & $272(580)$ \\
Gly (POU) & Ser (POU) & C-terminal & $342(650)$ \\
\hline
\end{tabular}

a The chemical nature of each amino acid is indicated in parenthesis. HNP, hydrophobic nonpolar; POU, polar uncharged; +, basic; -, acidic.

b Positions of the changed amino acids corresponding to the Plum pox virus HC-Pro protein and the viral polyprotein (in parenthesis). 
CA). Northern blot analyses were performed with $10 \mu \mathrm{g}$ of total RNA, essentially as described previously. Green fluorescent protein (GFP) and HC-Pro mRNAs were detected with DIG-labeled riboprobes complementary to either GFP or HC-Pro sequences. Total RNA for analysis of short interfering RNAs (siRNAs) was extracted with Trizol (Invitrogen, Carlsbad, CA). Low molecular weight (LMW) RNA was further purified using the DNA/RNA Midi Kit (Qiagen). Twenty micrograms of LMW RNA was analyzed with a ${ }^{32} \mathrm{P}$-labeled GFP probe produced by random priming as described in Tenllado et al. (25).

Viral proteins were analyzed by western blot as described in Gonzalez-Jara et al. (6). HC-Pro was detected with a polyclonal antiserum (1:300 dilution) produced in our laboratory by immunization of a rabbit with purified HC-Pro protein expressed in Escherichia coli. PVX CP was detected with a monkey polyclonal antiserum (Amersham Biosciences, Rainham, UK) (1:300 dilution). PPV CP was detected with a rabbit polyclonal antiserum (1:2,000 dilution) (Instituto Valenciano de Investigaciones Agrarias [Valencia Institute for Agricultural Research] IVIA). An appro-

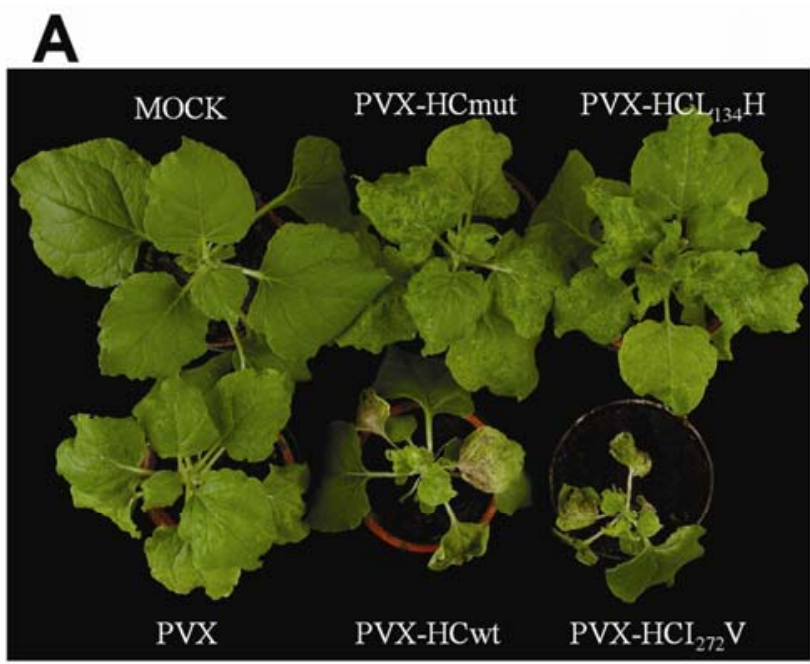

B

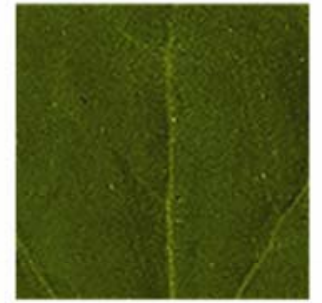

MOCK

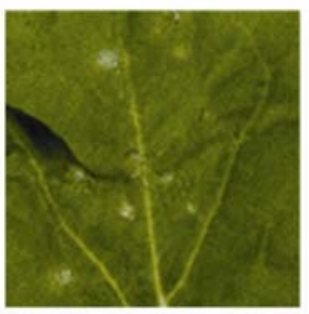

PVX-HCwt

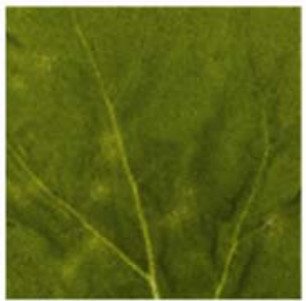

PVX

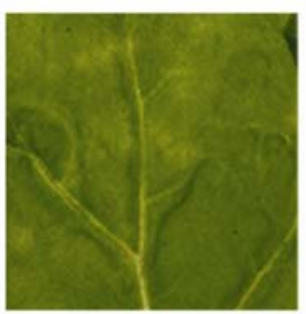

PVX-HCmut
Fig. 1. Characteristic symptoms induced in Nicotiana benthamiana plants by infection with Potato virus $X$ (PVX) or the different PVX recombinant viruses. A, Plants infected with PVX, PVX-HCwt, PVX-HCI ${ }_{272}$ V, PVXHcmut, or PVX-HCL ${ }_{134} \mathrm{H}$ compared with a mock-control at 18 days postinoculation (dpi). B, Detached inoculated leaves of plants infected with PVX, PVX-HCwt, or PVX-HCmut compared with a mock-control at $11 \mathrm{dpi}$. priate antibody conjugated with peroxidase was used in each case. Detection was performed using the enhanced chemiluminescence system (Amersham Biosciences).

Viral RNAs and proteins in doubly infected plants were analyzed as described in Gonzalez-Jara et al. (6).

\section{RESULTS}

Synergism with PVX recombinant viruses expressing HCPro of PPV. Using PVX as a vector, we tested the induction of viral synergism mediated by various cloned forms of PPV HCPro. p3.3HCwt contained a HC-Pro gene identical to the master sequence obtained by sequencing 14 HC-Pro cDNA products amplified by RT-PCR from the RNA extracts of plants infected with PPV isolate 5.15 or 3.3. p5.15HCmut contained a variant of the HC-Pro gene differing at 10 nucleotide positions with respect to that encoded by p3.3HCwt. These changes resulted in five amino acid substitutions in the HC-Pro protein: two in the N-terminal region, another two in the central region, and one in the $\mathrm{C}$-terminal region (Table 1). Clone p5.15HCmut apparently derived from a virus variant present as a minor fraction (one out of nine clones) of the 5.15 population. However, we cannot discard that it could have been generated during the cloning procedure, albeit special care to discard RT-PCR mistakes were undertaken, including a high-fidelity DNA polymerase.

To determine whether the expression of HC-Pro contained in p5.15HCmut could enhance the pathogenicity of PVX, HC-Pro sequences present in $\mathrm{p} 5.15 \mathrm{HCmut}$ or $\mathrm{p} 3.3 \mathrm{HCwt}$ were inserted into PVX to produce PVX-HCwt and PVX-HCmut. Four N. benthamiana plants were inoculated with RNA transcripts derived from each of the recombinant viruses or the empty PVX vector as a control. By 6 dpi, the control plants had developed vein clearing and chlorotic mosaic symptoms on systemically infected leaves (Fig. 1A). As expected, PVX-HCwt induced leaf curling and severe vein clearing by 7 to $8 \mathrm{dpi}$, followed by the necrosis of systemically infected leaves and stems, and usually caused plant death during the course of the following few days. In contrast, symptoms induced by PVX-HCmut were much milder; although the plants developed initial vein clearing followed by a mild chlorotic mosaic, they never showed necrosis. Differences in viral symptoms were also observed in inoculated leaves; PVX-HCwt produced necrotic spots, while PVX-HCmut produced chlorotic spots similar to those induced by PVX (Fig. 1B).

Since the increase in PVX pathogenicity and viral accumulation during PVX-potyvirus interactions was mediated by the $\mathrm{HC}$ Pro protein $(7,20,26,27)$, the accumulation of this in plants was analyzed by western blotting. Proteins were extracted from the second systemically infected half-leaf at 10 dpi when leaves manifested vein clearing symptoms. This assay showed that HC-
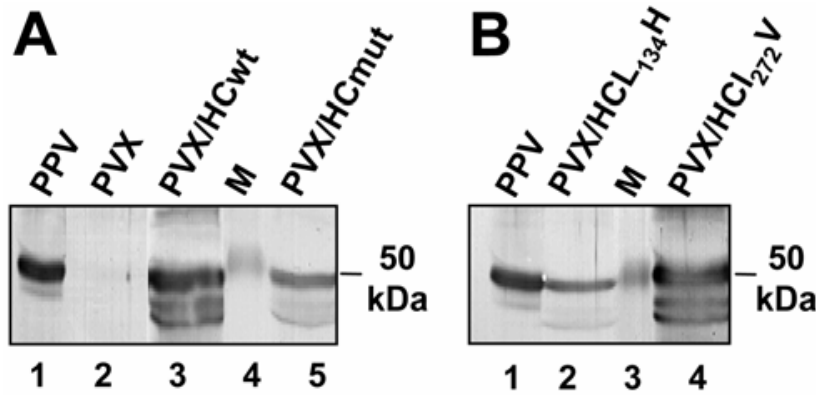

Fig. 2. Western blot analysis showing accumulation of Plum pox virus (PPV) helper component-proteinase (HC-Pro) in Nicotiana benthamiana plants infected with recombinant viruses $\mathbf{A}$, PVX-HCwt or PVX-HCmut and $\mathbf{B}$, PVX-HCL ${ }_{134} \mathrm{H}$ or PVX-HCI ${ }_{272}$ V. A sample from a plant infected with PPV was loaded to provide an accurate control of the putative size of HC-Pro. M, Prestained molecular weight marker. Data shown are composite images from noncontiguous lanes from a single blot. 
Pro accumulated in plants infected with PVX-HCmut, although a moderate reduction in the level of protein accumulated was seen compared with PVX-HCwt-infected plants (Fig. 2A, lanes 5 and 3).

PVX recombinants carrying foreign sequences can lose these during systemic infection owing to recombination between the artificially duplicated and the native PVX CP promoters (4). Therefore, the stability of the viral progenies was assayed by northern blotting using two kinds of probes, one complementary to PVX CP sequences, and the other complementary to PPV HCPro. Total RNAs were extracted from systemically infected halfleaves remaining after sampling for protein analysis. As shown in Figure $3 \mathrm{~A}$ and B, genomic (g)RNA from both the PVX-HCwt and the PVX-HCmut recombinant viruses retained the insert to similar extents at this time point, indicating that the lesser accumulation of HC-Pro in plants infected with PVX-HCmut was probably not related to insert instability. Additionally, and in agreement with data obtained from the protein analysis, accumulation of HC-Pro, triple-gene-block (TGB), and CP subgenomic (sg)RNAs in plants infected with PVX-HCmut was sixfold less than in plants infected with PVX-HCwt (Fig. 3A and B, compare lanes 5 and 4). Thus, the lack of a synergistic response in the plants plus a reduction in the level of PVX sgRNAs accumulation was associated with a mutated variant of the HC-Pro from PPV. Remarkably, neither PVX-HCmut nor PVX-HCwt accumulated gRNA to higher extents than the empty PVX vector, arguing against an overall higher accumulation of PVX as the main cause of symptom exacerbation in viral synergism.

A single amino acid substitution in the central domain of PPV HC-Pro prevents HC-Pro from mediating an increase in PVX pathogenicity. It has been reported that the loss of the ability of HC-Pro to induce an increase in PVX pathogenicity is connected to mutations located in the central region of the protein (24). In the present study, two of the five amino acid differences between PVX-HCwt and PVX-HCmut in the HC-Pro gene were localized within the central region of the protein (leucine to histidine and isoleucine to valine at positions 134 and 272, respectively; Table 1). To check whether any of these mutations were involved in the abolishment of the capacity of HC-Pro to induce synergism, mutations $\mathrm{L}_{134} \mathrm{H}$ and $\mathrm{I}_{272} \mathrm{~V}$ were reproduced individually in PVX-HCwt, yielding PVX- $\mathrm{HCL}_{134} \mathrm{H}$ and $\mathrm{PVX}-\mathrm{HCI}_{272} \mathrm{~V}$. Four $N$. benthamiana plants were inoculated with RNA transcripts from each of the new recombinant viruses, as well as with transcripts from PVX, PVX-HCwt, or PVX-HCmut as controls. From 7 to 8 dpi onward, PVX- $\mathrm{HCI}_{272} \mathrm{~V}$-inoculated plants developed local necrotic spots and severe systemic vein clearing, followed by necrosis of leaves and stems, resembling symptoms induced by PVX-HCwt (Fig. 1). In contrast, all the plants infected with PVX-HCL ${ }_{134} \mathrm{H}$ showed symptoms similar to those of plants
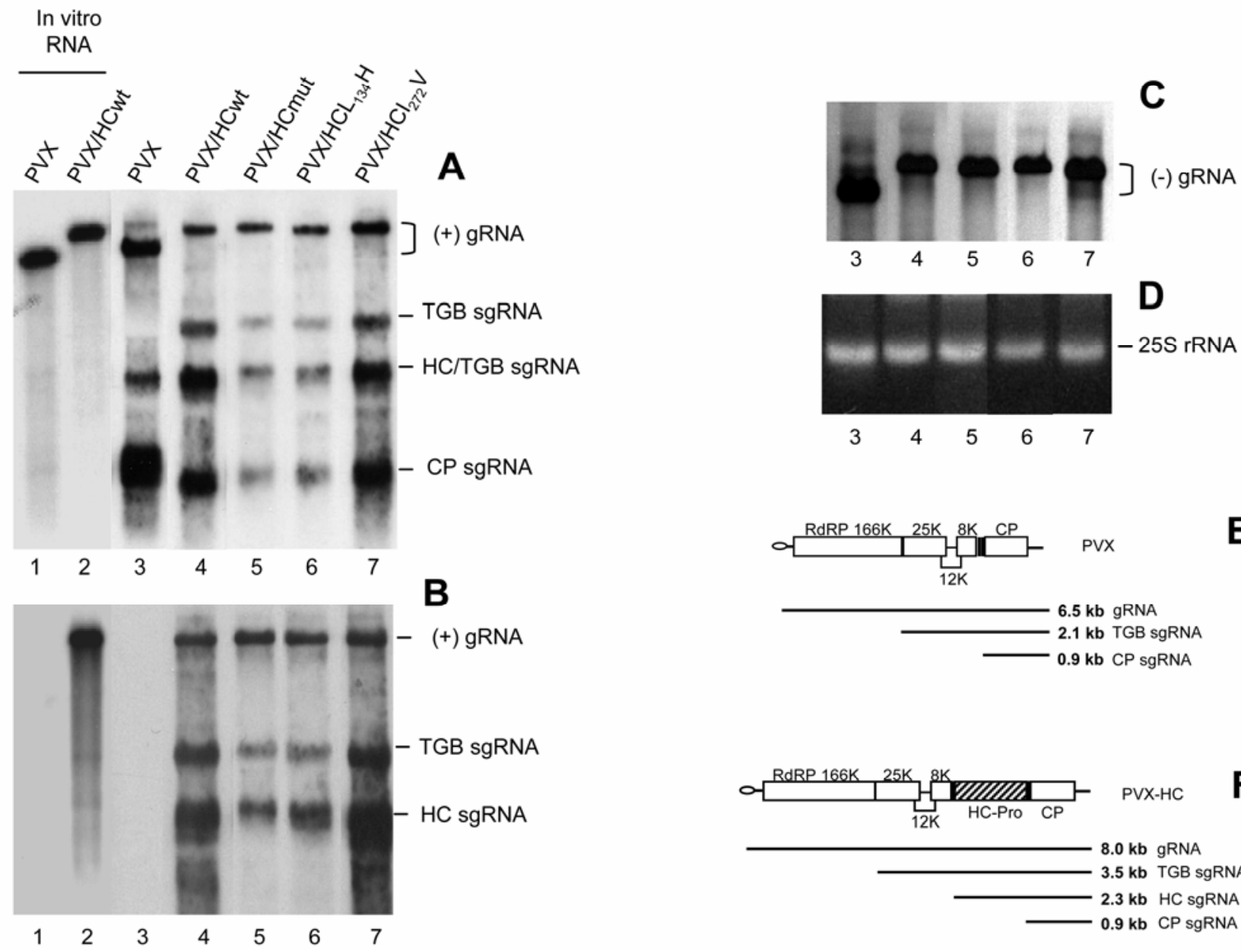

$\mathbf{E}$

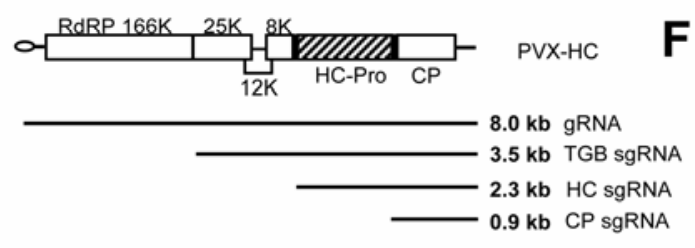

Fig. 3. Northern blot analysis of total RNA extracts of Nicotiana benthamiana plants infected with Potato virus $X$ (PVX) or with PVX recombinant viruses PVXHCwt, PVX-HCmut, PVX-HCL ${ }_{134} \mathrm{H}$, or PVX-HCI 272 V. Total RNA was hybridized with a probe complementary to A, PVX (+) strand RNA; B, helper componentproteinase (HC-Pro) sequence; or C, PVX (-) strand RNA. The northern blots in A and B were performed using $2 \mu \mathrm{g}$ of total RNA. PVX genomic (g)RNA and the major subgenomic (sg)RNAs, triple-gene-block (TGB) sgRNA and coat protein sgRNA (plus HC-Pro sgRNA in the case of the recombinant viruses) were detected. Note the different sizes of viral RNAs (gRNA and TGB sgRNA) from PVX compared with RNAs from the recombinant viruses. The northern blot in C was performed by loading $6 \mu \mathrm{g}$ of total RNA. D, Equal loading of RNA samples in A was assessed by ethidium bromide staining of agarose gels. Data shown are composite images from noncontiguous lanes from a single blot. In vitro-transcribed PVX and PVX/HCwt RNA used in the inoculum were run for comparison. Diagrams show genome organization and approximate sizes of viral RNAs for $\mathbf{E}$, PVX and F, PVX recombinant viruses carrying HC-Pro variants. 


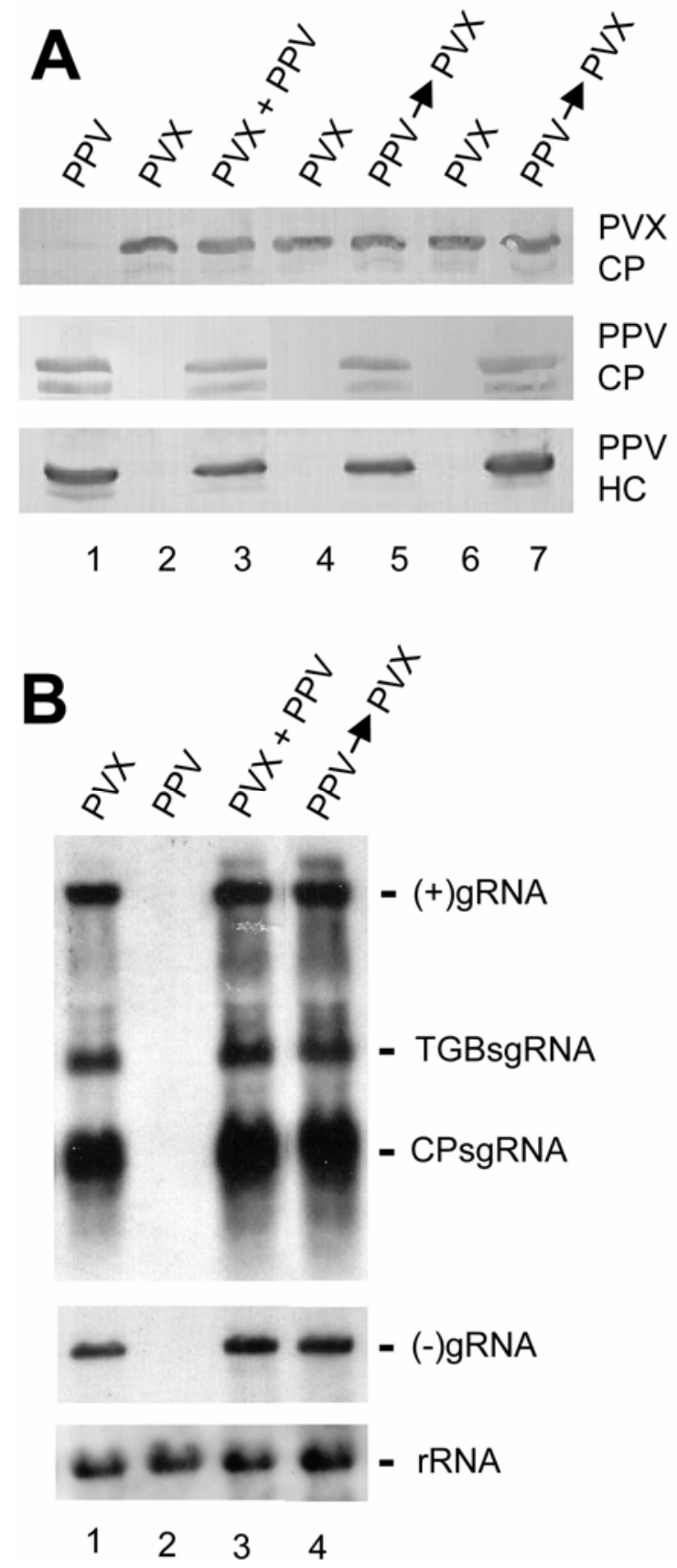

Fig. 4. Viral accumulation in Nicotiana benthamiana plants doubly infected with Potato virus $X$ (PVX) and Plum pox virus (PPV). A, Western blot analysis showing the accumulation of viral proteins in plants simultaneously or sequentially inoculated with PVX and PPV. Plants were inoculated with a combination of PVX+PPV (lane 3), or first with PPV and then 12 or $24 \mathrm{~h}$ later with PVX in opposite half-leaves (lanes 5 and 7, respectively). As controls, plants were inoculated with PPV alone (lane 1), PVX alone (lane 2), or singly inoculated with PVX at the time when this virus was sequentially inoculated with PPV (lane 4, $12 \mathrm{~h}$; lane 6, $24 \mathrm{~h}$.). Total proteins were immunoprobed using antiserum specific for PVX coat protein (CP) (upper panel), PPV CP (middle panel), or PPV helper component-proteinase (lower panel). B, Northern blot analysis of total RNA extracts of plants infected with PVX alone or in combination with PPV. Total RNA was hybridized with a probe complementary to PVX (+) strand RNA (upper panel) or PVX (-) strand RNA (middle panel). PVX $(+)$ strand RNA detection was performed using $2 \mu \mathrm{g}$ of total RNA. PVX genomic (g)RNA and the two major subgenomic (sg)RNAs, triple-gene-block sgRNA and CP sgRNA, are indicated. PVX (-) strand RNA detection was performed using $6 \mu \mathrm{g}$ of total RNA. The loading of similar amounts of total RNA was assessed using a digoxigenin-labeled probe specific for 18S ribosomal RNA (lower panel). Data shown are composite images from noncontiguous lanes from a single blot. Lane 1, PVX; 2, PPV; 3, PVX+PPV; and 4, PVX+PPV (with a 24-h lag period). infected with PVX-HCmut or PVX: local chlorotic spots and a mild systemic chlorotic mosaic. This suggests that the single amino acid substitution $\mathrm{L}_{134} \mathrm{H}$ in the central region of PPV HCPro is responsible for rendering the protein incapable of inducing a synergistic response upon PVX-HC infection.

Again, HC-Pro accumulation was greater in plants infected with the mutant recombinant virus that induced the synergistic response $\left(\mathrm{PVX}-\mathrm{HCI}_{272} \mathrm{~V}\right)$ than in plants infected with the defective $\mathrm{HC}$-Pro expressing recombinant virus (PVX-HCL ${ }_{134} \mathrm{H}$ ) (as assayed by western blot) (Fig. 2B, compare lanes 4 and 2). Northern blot hybridizations confirmed the stability of the viral progenies and the data obtained from the protein analysis. Not only was HC-Pro sgRNA accumulation greater but so too was that of TGB and CP sgRNA (four- to sixfold) in plants infected with PVX$\mathrm{HCI}_{272} \mathrm{~V}$ compared with plants infected with $\mathrm{PVX}-\mathrm{HCL}_{134} \mathrm{H}$ (Fig. $3 \mathrm{~A}$ and $\mathrm{B}$, compare lanes 7 and 6 ). The stability of point mutations $\mathrm{L}_{134} \mathrm{H}$ or $\mathrm{I}_{272} \mathrm{~V}$ in viral progenies was confirmed by sequencing the amplified HC-Pro sequence obtained from total RNA extracts by RT-PCR. Moreover, no other nucleotide changes were seen in the HC-Pro region. The possibility that mutations other than $\mathrm{L}_{134} \mathrm{H}$ or $\mathrm{I}_{272} \mathrm{~V}$ were connected with the phenotype shown by plants infected with either of the mutant recombinant viruses was therefore rejected.

It is worth noting that PVX (+) strand gRNA seemed to accumulate at roughly similar levels independently of whether the plants were infected with PVX recombinant viruses that induced the synergistic response (PVX-HCI ${ }_{272} \mathrm{~V}$ and PVX-HCwt) or with those that did not (PVX-HCL ${ }_{134} \mathrm{H}$ and $\mathrm{PVX}-\mathrm{HCmut}$ ) (differences were less than 1.5-fold; Fig. 3A and B). The enhancement of symptoms during the interaction of PVX with potyviruses correlates with a disproportionate increase in the accumulation of PVX (-) strand RNA compared with PVX (+) strand RNA in tobacco (27) and a slight increase in N. benthamiana (6). Therefore, levels of PVX (-) strand RNA in plants infected with the different PVX recombinant viruses were examined. Remarkably, the PVX (-) strand gRNA accumulated at roughly comparable levels in plants infected with either of the recombinant viruses, independently of whether they induced a synergistic response or not (Fig. 3C). Altogether, these results indicated that PPV HC-Pro variants capable of inducing a synergistic response in $N$. benthamiana enhance and/or stabilize PVX sgRNAs but not PVX (+) and (-) gRNA.

Synergism in PVX/PPV doubly infected plants. Northern blot hybridizations showed that the accumulation of PVX $(+)$ and (-) gRNA in $N$. benthamiana plants infected with PVX recombinant viruses expressing HC-Pro did not increase over that of plants infected with PVX, despite the enhancement of symptoms observed in some recombinants (Fig. 3A and C). Since the insertion of a foreign sequence into the PVX vector could have had a debilitating effect on virus multiplication, the characteristics of PVX/PPV interaction in $N$. benthamiana were investigated in a series of double infection experiments performed with native viruses. Taken into account that the maximum enhancement of PVX synthesis by potyviruses in coinfected tobacco plants depends on the timing of viral invasion (7), the effect of virus inoculation time on PVX accumulation was examined in a series of sequential inoculations. PVX was inoculated into plants either simultaneously with, or 12 to $24 \mathrm{~h}$ after, inoculating PPV into the same leaves. Control plants infected with PVX alone developed the expected phenotype, while the PPV-infected plants showed typically mild vein clearing and systemic, chlorotic mosaic. However, those inoculated with PVX and PPV at the same time, or separated by a 12- to 24-h lag period, showed a synergistic reaction comparable to that observed in plants infected with PVX recombinants expressing functional PPV HC-Pro. This led to vein clearing and necrosis of the systemically infected leaves and eventually caused the death of the plants by $14 \mathrm{dpi}$ (data not shown).

To determine whether the increase in symptom severity in $N$. benthamiana plants coinfected with PVX and PPV correlated 
with changes in PVX accumulation, the level of PVX CP in these plants was analyzed by western blotting (Fig. 4A, upper panel). PVX CP levels did not increase in doubly infected plants compared with singly infected controls. Moreover, accumulation of PVX CP did not vary among the different groups of plants, regardless of whether they had been inoculated with PVX and PPV simultaneously or separated by 12 to $24 \mathrm{~h}$. The accumulation of PPV CP and HC-Pro was similar in both singly or doubly infected plants, as reported in other potyvirus synergistic interactions (Fig. 4A, middle and bottom panels).

In agreement with the results obtained for PVX CP detection, the accumulation of PVX (+) and (-) strand RNA in the doubly infected plants was similar to that observed in their singly infected counterparts, as determined by northern blotting (Fig. 4B). Moreover, PVX RNAs accumulated in plants coinoculated with PVX and PPV at levels comparable to those in plants inoculated first with PPV and then with PVX $24 \mathrm{~h}$ later. Thus, the failure of PPV to enhance PVX CP and RNA accumulation in doubly infected $N$. benthamiana plants does not depend on the timing of virus infection. When PPV RNA accumulation was examined in these same plants, PPV (+) strand RNA levels were not different in singly or doubly infected plants (data not shown). Altogether these results indicate that the PVX titer does not vary during the interaction of PVX and PPV in $N$. benthamiana, despite the enhancement of symptoms.

PPV HC-Pro carrying the mutation $\mathrm{L}_{134} \mathrm{H}$ shows impaired PTGS suppression. The synergism induced by HC-Pro is thought to be mediated by its capability to suppress plant antiviral defenses based on PTGS (29). Therefore, we tested the synergismdefective mutant $\mathrm{HC}$-Pro $\mathrm{L}_{134} \mathrm{H}$ to determine whether its ability to suppress gene silencing was different form that of HC-Pro wt. Agrobacterium-based delivery of a cDNA construct encoding functional GFP was used as the silencing system. Transient expression of GFP reaches its maximum 2 to 3 days after infiltration and then decays progressively due to PTGS, while coexpression with a PTGS suppressor keeps GFP expression at a high level for longer (10). The GFP construct was coinfiltrated in N. benthamiana leaves with $\mathrm{HC}$-Pro wt (HCwt), HC-Pro $\mathrm{L}_{134} \mathrm{H}$ $\left(\mathrm{HCL}_{134} \mathrm{H}\right.$ ), or a GUS gene that served as negative control (Fig. 5A). Patches infiltrated with GFP plus GUS showed slight luminescence under UV light 2 to 3 days after infiltration that did not increase further or even faded progressively. GFP plus $\mathrm{HCwt}$ showed considerably stronger luminescence that continued to increase at least until day 9. In contrast, GFP plus $\mathrm{HCL}_{134} \mathrm{H}$ showed slight luminescence comparable to that observed in GFP plus GUS-infiltrated patches. Northern blots analysis of RNA extracted from the infiltrated patches confirmed these visual observations (Fig. 5B). In patches coinfiltrated with GFP and GUS, GFP mRNA had accumulated at high levels by 3 days postinfiltration but decreased considerably by days 5 and 7 . In contrast, GFP coinfiltrated with HCwt showed high GFP mRNA levels until day 7. GFP coinfiltrated with $\mathrm{HCL}_{134} \mathrm{H}$ showed essentially the same GFP mRNA kinetics as the GFP plus GUS combination. Correspondingly, GFP-derived siRNAs, a hallmark of PTGS, accumulated to similar extents in GFP plus GUS-infiltrated tissues and in GFP plus $\mathrm{HCL}_{134} \mathrm{H}$-infiltrated tissues, while they accumulated fivefold less in those infiltrated with GFP plus HCwt (Fig. 5C). These results show that $\mathrm{HCL}_{134} \mathrm{H}$ is impaired in its ability to suppress PTGS of a sense transgene used as a silencing locus.

The inability of $\mathrm{HCL}_{134} \mathrm{H}$ to suppress silencing in the transient expression assay might be attributable to an instability of the protein. Thus, the accumulation of transiently expressed HCwt and $\mathrm{HCL}_{134} \mathrm{H}$ was tested in protein extracts from the same coinfiltrated tissues used for GFP mRNA analysis (Fig. 6A). Accumulation of HCwt was slightly stronger than that of $\mathrm{HCL}_{134} \mathrm{H}$ at 3 days. At 5 days the difference in accumulation was more marked. It has been described that transient expression of mutant variants of TEV HC-Pro is posttranscriptionally silenced when the mutation affects its capability to suppress PTGS (11). To test this, we analyzed HC-Pro mRNA accumulation in HCwt- and $\mathrm{HCL}_{134} \mathrm{H}$ infiltrated tissues. As expected, HCwt mRNA accumulated to large amounts by 3 or 5 days after infiltration, with levels decreasing slightly by day 7 . However, $\mathrm{HCL}_{134} \mathrm{H}$ mRNA accumulated less by day 3 and decreased severely after 5 days (Fig. 6B). This strongly suggests that $\mathrm{HCL}_{134} \mathrm{H}$ mRNA is targeted for degradation due to the incapacity of the mutant protein to suppress PTGS.

\section{DISCUSSION}

The PVX recombinant virus expressing the wild-type variant of PPV HC-Pro induced the expected enhancement of PVX pathogenicity (manifested as necrosis and plant death), while the
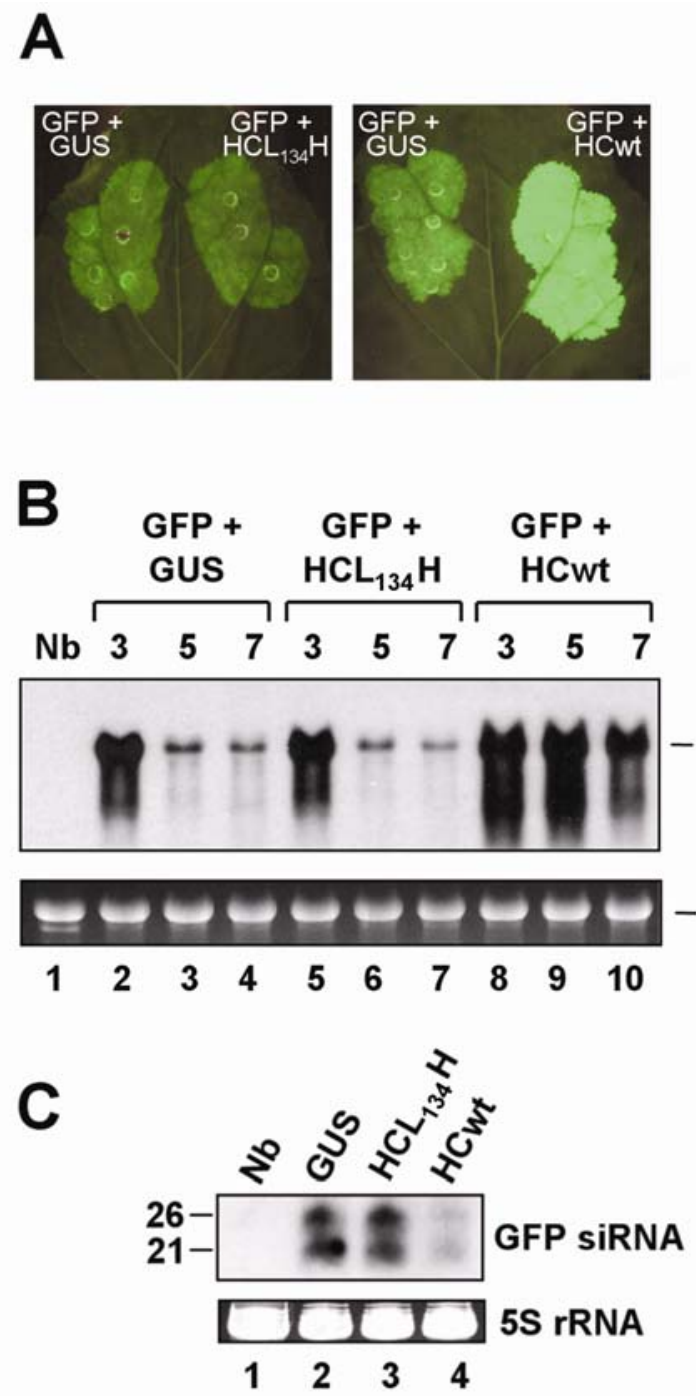

Fig. 5. Expression of Plum pox virus $\mathrm{HCL}_{134} \mathrm{H}$ protein in Nicotiana benthamiana failed to suppress posttranscriptional gene silencing of the green fluorescent protein (GFP) transgene. A, Leaves were infiltrated with combinations of Agrobacterium cultures containing GFP plus either GUS, $\mathrm{HCL}_{134} \mathrm{H}$, or HCwt, as indicated. Photographs were taken at 9 days after infiltration. B, Northern blot analysis of total RNA extracted from agroinfiltrated patches such as those shown in $\mathbf{A}$ at 3, 5, and 7 days after infiltration. Total RNA $(10 \mu \mathrm{g})$ was hybridized with a probe complementary to GFP. The RNA loaded in lane $\mathrm{Nb}$ came from a noninfiltrated leaf. Ethidium bromide staining of $25 \mathrm{~S}$ rRNA is shown as loading control. $\mathbf{C}$, Accumulation of GFP short interfering RNAs (siRNAs) at 7 days after agroinfiltration. Low molecular weight RNA was fractionated in $15 \%$ polyacrylamide gels containing $7 \mathrm{M}$ urea, and GFP siRNAs were detected using a random-primed ${ }^{32} \mathrm{P}$-labeled probe. The sizes of the DNA markers are indicated. Ethidium bromide staining of $5 \mathrm{~S}$ rRNA is shown as a loading control. 
recombinant virus PVX-HCmut expressing a variant of PPV HCPro with five amino acid substitutions was unable to induce this. Experiments using PVX recombinants encoding mutant variants of HC-Pro containing single point mutations $\mathrm{L}_{134} \mathrm{H}$ or $\mathrm{I}_{272} \mathrm{~V}$ demonstrated that mutation $\mathrm{L}_{134} \mathrm{H}$ alone was sufficient to render $\mathrm{HC}$ Pro protein defective for synergism. This result was consistent with the fact that mutation $\mathrm{L}_{134} \mathrm{H}$ implies drastic changes in the chemical properties of the amino acid affected, while $\mathrm{I}_{272} \mathrm{~V}$ does not. It is noteworthy that other potyviruses such as Potato virus A, Tobacco vein mottling virus, Zucchini yellow mosaic virus, PVY, and TEV show either the same residue or an amino acid of similar chemical characteristics at equivalent positions in their genomes. This could make the results described applicable to these other potyviruses.

The HC-Pro of PPV was shown to have PTGS suppressor activity in the transient silencing suppression assay. In contrast, the $\mathrm{HCL}_{134} \mathrm{H}$ mutant showed no such activity. These results show that the same single point mutation that impaired PPV HC-Pro's ability to suppress PTGS also abolished its ability to induce synergism. This supports the link between these two functions in potyvirus HC-Pro. Lots of data are available on mutations of HC-Pro that correlate the suppression of PTGS with genome amplification, long-distance movement, and symptom modulation in potyviruses $(11,22)$. However, data correlating synergism capability and the suppression of PTGS by potyviral HC-Pro are much less abundant and restricted to the combination TEV-tobacco (1). Evidence for this came from a transgenic line expressing mutant HC-Pro sequences that failed to develop synergistic disease when infected with PVX, and which failed to suppress silencing in the offspring of a transgenic line silenced for a reporter gene. Never-
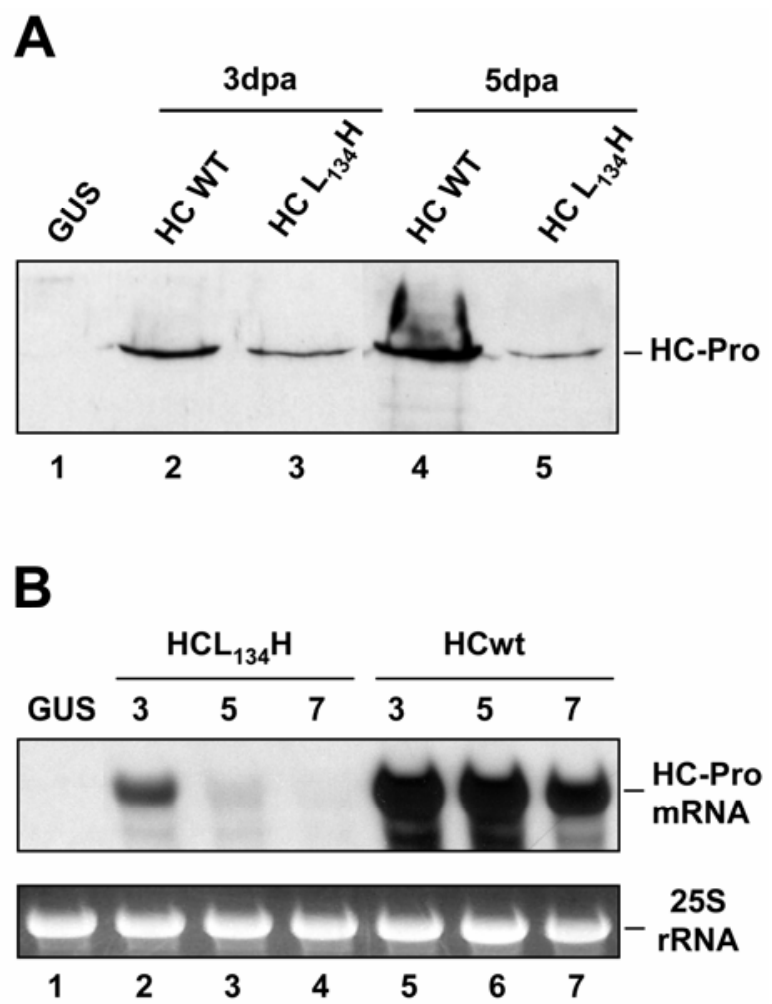

Fig. 6. Accumulation of transiently expressed $\mathrm{HCwt}$ and $\mathrm{HCL}_{134} \mathrm{H}$ in agroinfiltrated tissue of Nicotiana benthamiana. A, Western blot analysis of $\mathrm{HCwt}$ and $\mathrm{HCL}_{134} \mathrm{H}$ in protein extracts from the same coinfiltrated leaves used for green fluorescent protein (GFP) mRNA analysis shown in Figure 5, at 3 and 5 days after infiltration. Proteins loaded in lane GUS came from a leaf infiltrated with GFP plus GUS constructs and served as a negative control. B, Northern blot analysis of $\mathrm{HCwt}$ and $\mathrm{HCL}_{134} \mathrm{H}$ mRNAs at 3, 5, and 7 days after infiltration. The RNA loaded in lane GUS came from a leaf infiltrated with GFP plus GUS constructs. theless, the homozygous TEV-K line used in the genetic crosses no longer accumulated detectable levels of HC-Pro protein, precluding any unequivocal inferences to be drawn. The present paper confirms that the suppression of PTGS and the ability to induce a synergistic response in $N$. benthamiana are closely associated in PPV HC-Pro.

Recently, Plisson and coworkers (19) reported the partial characterization of the structure of HC-Pro from Lettuce mosaic virus, establishing the basis upon which to study the relationships between structure and the biochemical roles of HC-Pro protein. Domain 1 of HC-Pro, in which mutation $\mathrm{L}_{134} \mathrm{H}$ occurs, as well as the hinge domain, have been hypothesized to contain the active sites needed for several related functions such as genome amplification, long-distance movement, suppression of gene silencing, and synergism. What is still undetermined is whether these functions are enclosed in the same or different active sites, and how host and/or viral factors interact with them. Thus, it is possible that point mutation $\mathrm{L}_{134} \mathrm{H}$ precisely affects an active site of the protein required for synergism and the suppression of gene silencing. Another explanation for the dysfunction of $\mathrm{HCL}_{134} \mathrm{H}$ is that point mutation $\mathrm{L}_{134} \mathrm{H}$ may affect the conformation/stability of the protein. Instability of the mutant protein, however, is unlikely to account for the observed phenotypes since Agrobacteriummediated transient expression of $\mathrm{HCL}_{134} \mathrm{H}$ led to levels of mutant protein roughly similar to those induced by wild-type HC-Pro before the onset of RNA silencing.

The synergistic effect on disease development induced by PVX expressing functional HC-Pros (PVX-HCwt and $\mathrm{PVX}-\mathrm{HCI}_{272} \mathrm{~V}$ ) did not lead to increased PVX gRNA levels over those of plants infected with the empty PVX vector or PVX recombinants expressing HC-Pro variants that did not induce synergism (PVXHCmut and PVX-HCL $\left.{ }_{134} \mathrm{H}\right)$. Similar results have been reported by others in $N$. benthamiana infected with PVX or PVX expressing HC-Pro derived from PVY or TEV $(3,20)$. However, Yang and Ravelonandro (31) reported a twofold enhancement of PVX accumulation in this host based on the comparison of infections by PVX or PVX expressing the HC-Pro from PPV. Besides some minor divergences in $N$. benthamiana, the most apparent discrepancy with regard to PVX accumulation is seen when comparing data obtained from $N$. tabacum infected with native viruses (where PVX levels are 10-fold higher than in single infection) $(26,27)$. The data obtained from infections of $N$. benthamiana with PVX recombinant viruses could lead to misinterpretations since the use of vectors containing foreign sequences might lead to the attenuation of virus accumulation. However, as we recently showed, the explanation is probably much more related to the differential response of these two Nicotiana spp. to mixed infections. This indicates that PVX titer enhancement was host-dependent (6). Indeed, the double infection experiments with PPV and PVX reported here show that the enhanced pathogenicity associated with synergistic interaction in $N$. benthamiana is not the consequence of more efficient PVX replication. Other mechanisms must therefore be involved in the induction of synergism (described below).

The lack of a drastic enhancement in PVX titers during PVXpotyvirus synergistic interactions in $N$. benthamiana seems to indicate that there is a threshold above which viral accumulation cannot increase in this host, despite inhibition of antiviral defenses by HC-Pro. However, this model does not fit well with the lesser accumulation of sgRNAs observed with PVX recombinants expressing HC-Pro variants that do not induce synergism. The insertion of a foreign gene into the PVX genome may somehow be detrimental to virus fitness. However, the presence of an active HC-Pro might compensate for this, restoring sgRNAs levels to their maximum.

Potyviral HC-Pro can interfere with miRNAs-guided mRNA regulation and promotes developmental abnormalities in plants $(5,12)$. In addition, p25 encoded by PVX has been shown to func- 
tion as a viral suppressor of gene silencing (30). Moreover, HCPro and p25 interfere with the PTGS pathway at two different steps $(14,18,30)$. Synergistic symptoms manifested in plants might be provoked by the combined action of these viral suppressors on multiple RNA silencing pathways. Therefore, HC-Pro from potyviruses might have a role in the augmented disease phenotype observed in PVX-potyvirus synergistic interactions that is not entirely linked to its involvement in the enhancement of PVX accumulation observed in some hosts.

\section{ACKNOWLEDGMENTS}

We thank A. G. von Arnim (University of Tennessee, Knoxville) for providing pAVA393, and J. C. Carrington (Center for Gene Research and Biotechnology, Oregon State University) for providing the pSLJ-GFP clone. P. González-Jara, F. A. Atencio, and D. Barajas were supported by doctoral fellowships from MECD-FPI (Spain), CONICET (Argentina), and the Comunidad de Madrid/MECD-FPU (Spain), respectively. B. Martínez-García is the recipient of a postdoctoral contract from the Comunidad de Madrid and the CSIC (Spain). This work was supported by grants BIO2003-03428 from the CICYT-MCyT and 07G/0043/2003 from the Comunidad de Madrid (Spain).

\section{LITERATURE CITED}

1. Anandalakshmi, R., Pruss, G. J., Ge, X., Marathe, R., Mallory, A. C., Smith, T. H., and Vance, V. B. 1998. A viral suppressor of gene silencing in plants. Proc. Natl. Acad. Sci. USA 95:13079-13084.

2. Baulcombe, D. C., Chapman, S., and Santa-Cruz, S. 1995. Jellyfish green fluorescent protein as a reporter for virus infections. Plant J. 7:1045-1053.

3. Brigneti, G., Voinnet, O., Li, W. X., Ji, L. H., Ding, S. W., and Baulcombe, D. C. 1998. Viral pathogenicity determinants are suppressors of transgene silencing in Nicotiana benthamiana. EMBO J. 17:67396746.

4. Chapman, S., Kavanagh, T., and Baulcombe, D. C. 1992. Potato virus X as a vector for gene expression in plants. Plant J. 2:549-557.

5. Dunoyer, P., Lecellier, C. H., Parizotto, E. A., Himber, C., and Voinnet, O. 2004. Probing the microRNA and small interfering RNA pathways with virus-encoded suppressors of RNA silencing. Plant Cell 16:1235-1250.

6. Gonzalez-Jara, P., Tenllado, F., Martinez-Garcia, B., Atencio, F. A., Barajas, D., Vargas, M., Diaz-Ruiz, J., and Diaz-Ruiz, J. R. 2004. Hostdependent differences during synergistic infection by Potyviruses with potato virus X. Mol. Plant Pathol. 5:29-35.

7. Goodman, R. M., and Ross, A. F. 1974. Enhancement of potato virus X synthesis in doubly infected tobacco occurs in doubly infected cells. Virology 58:16-24.

8. Higuchi, R., Krummel, B., and Saiki, R. K. 1988. A general method of in vitro preparation and specific mutagenesis of DNA fragments: Study of protein and DNA interactions. Nucleic Acids Res. 16:7351-7367.

9. Hull, R. 2002. Matthews' Plant Virology. 4th ed. Academic Press, London.

10. Johansen, L. K., and Carrington, J. C. 2001. Silencing on the spot. Induction and suppression of RNA silencing in the Agrobacterium-mediated transient expression system. Plant Physiol. 126:930-938.

11. Kasschau, K. D., and Carrington, J. C. 2001. Long-distance movement and replication maintenance functions correlate with silencing suppression activity of potyviral HC-Pro. Virology 285:71-81.
12. Kasschau, K. D., Xie, Z., Allen, E., Llave, C., Chapman, E. J., Krizan, K. A., and Carrington, J. C. 2003. P1/HC-Pro, a viral suppressor of RNA silencing, interferes with Arabidopsis development and miRNA function. Dev. Cell 4:205-217.

13. Laín, S., Riechmann, J. L., and Garcia, J. A. 1989. The complete nucleotide sequence of plum pox potyvirus RNA. Virus Res. 13:157-172.

14. Llave, C., Kasschau, K. D., and Carrington, J. C. 2000. Virus-encoded suppressor of posttranscriptional gene silencing targets a maintenance step in the silencing pathway. Proc. Natl. Acad. Sci. USA 97:1340113406.

15. Logemann, J., Schell, J., and Willmitzer, L. 1987. Improved method for the isolation of RNA from plant tissue. Anal. Biochem. 163:16-20.

16. López-Moya, J. J., Canto, T., López-Abella, D., and Díaz-Ruíz, J. R. 1994. Differentiation of Mediterranean plum pox virus isolates by coat protein analysis. Plant Pathol. 43:164-171.

17. Marathe, R., Anandalakshmi, R., Smith, T. H., Pruss, G. L., and Vance, V. B. 2000. RNA viruses as inducers, suppressors and targets of post-transcriptional gene silencing. Plant Mol. Biol. 43:295-306.

18. Moissiard, G., and Voinnet, O. 2004. Viral suppression of RNA silencing in plants. Mol. Plant Pathol. 5:71-82.

19. Plisson, C., Drucker, M., Blanc, S., German-Retana, S., Le Gall, O., Thomas, D., and Bron, P. 2003. Structural characterization of HC-Pro, a plant virus multifunctional protein. J. Biol. Chem. 278:23753-23761.

20. Pruss, G., Ge, X., Shi, X. M., Carrington, J. C., and Vance, V. B. 1997. Plant viral synergism: The potyviral genome encodes a broad-range pathogenicity enhancer that transactivates replication of heterologous viruses. Plant Cell 9:859-868.

21. Roth, B. M., Pruss, G. J., and Vance, V. B. 2004. Plant viral suppressors of RNA silencing. Virus Res. 102:97-108.

22. Sáenz, P., Quiot, L., Quiot, J. B., Candresse, T., and Garcia, J. A. 2001. Pathogenicity determinants in the complex virus population of a plum pox virus isolate. Mol. Plant-Microbe Interact. 14:278-287.

23. Sambrook, J., Fritsch, E. F., and Maniatis, T. 1989. Molecular Cloning: A Laboratory Manual. Cold Spring Harbor Laboratory, Cold Spring Harbor, NY.

24. Shi, X. M., Miller, H., Verchot, J., Carrington, J. C., and Vance, V. B. 1997. Mutations in the region encoding the central domain of helper component-proteinase (HC-Pro) eliminate potato virus X/potyviral synergism. Virology 231:35-42.

25. Tenllado, F., Barajas, D., Vargas, M., Atencio, F. A., González-Jara, P., and Díaz-Ruíz, J. R. 2003. Transient expression of homologous hairpin RNA causes interference with plant virus infection and is overcome by a virus encoded suppressor of gene silencing. Mol. Plant-Microbe Interact. 16:149-158.

26. Vance, V. B. 1991. Replication of potato virus X RNA is altered in coinfections with potato virus Y. Virology 182:486-494.

27. Vance, V. B., Berger, P. H., Carrington, J. C., Hunt, A. G., and Shi, X. M. 1995. 5' Proximal potyviral sequences mediate potato virus X/potyviral synergistic disease in transgenic tobacco. Virology 206:583-590.

28. Vance, V. B., and Vaucheret, H. 2001. RNA silencing in plants-Defense and counterdefense. Science 292:2277-2280.

29. Voinnet, O. 2001. RNA silencing as a plant immune system against viruses. Trends Genet. 17:449-459.

30. Voinnet, O., Lederer, C., and Baulcombe, D. C. 2000. A viral movement protein prevents spread of the gene silencing signal in Nicotiana benthamiana. Cell 103:157-167.

31. Yang, S., and Ravelonandro, M. 2002. Molecular studies of the synergistic interactions between plum pox virus HC-Pro protein and potato virus X. Arch. Virol. 147:2301-2312. 